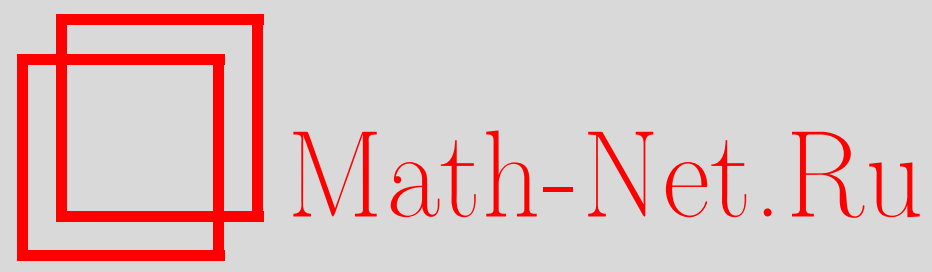

А. Ю. Карлович, Об индексе сингулярных интегральных операторов в рефлексивных пространствах Орлича, $M a$ тем. заметки, 1998, том 64, выпуск 3, 383-396

DOI: https://doi.org/10.4213/mzm1408

Использование Общероссийского математического портала Math-Net.Ru подразумевает, что вы прочитали и согласны с пользовательским соглашением http://www.mathnet.ru/rus/agreement

Параметры загрузки:

IP: 52.23 .180 .231

26 апреля 2023 г., 13:45:31

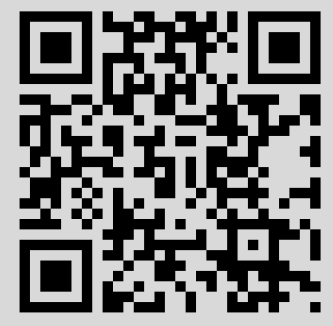




\section{ОБ ИНДЕКСЕ СИНГУЛЯРНЫХ ИНТЕГРАЛЬНЫХ ОПЕРАТОРОВ В РЕФЛЕКСИВНЫХ ПРОСТРАНСТВАХ ОРЛИЧА}

\section{А. Ю. Карлович}

Рассматривается банахова алгебра $\mathfrak{A}$ сингулярных интегральных операторов с матричными кусочно непрерьвными коэффициентами в рефлексивном пространстве Орлича $L_{M}^{n}(\Gamma)$. Предполагается, что $\Gamma$ принадлежит широкому подклассу кривых Карлесона, содержащему кривые с точками заострения (возврата), а также кривые типа логарифмической спирали. В данной работе получена формула для вычисления индекса произвольного оператора из алгебры $\mathfrak{A}$ в терминах его символа.

Библиография: 23 названия.

1. Введение. В работах И.Ц. Гохберга и Н.Я. Крупника начала 70-х годов получены критерии нётеровости и формулы для вычисления индекса операторов из банаховой алгебры, порожденной сингулярными интегральными операторами (СИО) с матричньми кусочно непрерывными коэффициентами в пространствах Лебега $L_{2}^{n}(\Gamma)[1]$ и $L_{p}^{n}(\Gamma, \rho)[2]$. Ограничения, которые накладывались на вес $\rho$ и кривую $\Gamma$ (рассматривался степенной вес $\rho$ и ляпуновская кривая $\Gamma$ ), были связаны с известными на то время достаточньми условиями ограниченности в пространствах Лебега $L_{p}(\Gamma, \rho), 1<p<\infty$, оператора сингулярного интегрирования с ядром Коши

$$
(S \varphi)(t):=\lim _{\varepsilon \rightarrow 0} \frac{1}{\pi i} \int_{\Gamma \backslash \Gamma(t, \varepsilon)} \frac{\varphi(\tau)}{\tau-t} d \tau, \quad t \in \Gamma,
$$

где $\Gamma(t, \varepsilon):=\{\tau \in \Gamma:|t-\tau|<\varepsilon\} . \Gamma$. Давид доказал [3], что оператор $S$ ограничен в пространстве $L_{p}(\Gamma), 1<p<\infty$, тогда и только тогда, когда Г является кривой Карлесона, т.е.

$$
\sup _{t \in \Gamma} \sup _{\varepsilon>0} \frac{1}{\varepsilon}|\Gamma(t, \varepsilon)|<\infty,
$$

где $|\Gamma(t, \varepsilon)|$ - мера порции $\Gamma(t, \varepsilon)$. Из этого результата и обзора [4] следует (см., например, [5], [6]), что для ограниченности оператора $S$ в весовом пространстве Лебега $L_{p}(\Gamma, \rho), 1<p<\infty$, необходимо и достаточно, чтобы вес $\rho$ удовлетворял условию Макенхаупта

$$
\sup _{t \in \Gamma} \sup _{\varepsilon>0}\left(\frac{1}{\varepsilon} \int_{\Gamma(t, \varepsilon)} \rho^{p}(\tau)|d \tau|\right)^{1 / p}\left(\frac{1}{\varepsilon} \int_{\Gamma(t, \varepsilon)} \rho^{-q}(\tau)|d \tau|\right)^{1 / q}<\infty
$$


где $1 / p+1 / q=1$. Согласно неравенству Гёльдера (3) влечет (2). Автором с использованием критерия Давида ограниченности оператора $S$ в пространствах $L_{p}(\Gamma)$ и интерполящии линейных операторов показано [7, теорема 3.6], что для ограниченности оператора $S$ в рефлексивном пространстве Орлича $L_{M}(\Gamma)$ также необходимо и достаточно, чтобы Г была кривой Карлесона.

Из леммы 3 работы Р.К. Сейфуллаева [8] следует, что для всех точек $t \in \Gamma$ кривая Карлесона удовлетворяет условию

$$
\arg (\tau-t)=O(-\log |\tau-t|), \quad \tau \rightarrow t \quad(\tau \in \Gamma)
$$

В работе А. Беттчера и Ю.И. Карловича [5] получен критерий нётеровости для операторов из банаховой алгебры СИО с кусочно непрерывными коэффициентами в пространстве Лебега $L_{p}(\Gamma, \rho)$, где $\rho$ - вес Макенхаупта, а $\Gamma$ - замкнутая жорданова кривая Карлесона, во всех точках $t \in \Gamma$ удовлетворяющая дополнительному условию

$$
\arg (\tau-t)=-\delta(t) \log |\tau-t|+O(1), \quad \tau \rightarrow t
$$

где $\delta: \Gamma \rightarrow \mathbb{R}$. В дальнейшем такие кривые будем называть логарифмическими кривыми Карлесона. В работе автора [7] при тех же предположениях относительно кривой Г получен критерий нётеровости для операторов из банаховой алгебры СИО с матричньми кусочно непрерывными коэффициентами в рефлексивном пространстве Орлича $L_{M}^{n}(\Gamma)$. Отметим также, что недавно в работах [6], [9] при изучении алгебры СИО в весовых пространствах Лебега ограничение (4) на кривую Карлесона было снято.

Для получения этих результатов широко использовались мощные современные средства изучения банаховых алгебр - теорема о двух проекторах [10], [11] и локальньй принцип Аллана-Дугласа (см., например, [12, разд. 1.34]). Эти средства позволяют построить символьное исчисление для банаховой алгебры, порожденной СИО с матричными кусочно непрерьвными коэффициентами, если до этого изучен "канонический” СИО со специальным скалярным кусочно непрерывным коэффициентом, ассоциированный с краевой задачей Римана. Однако эти методы не позволяют решить задачу о вычислении индекса оператора из указанной алгебры.

В настоящей работе, являющейся продолжением работы [7], вычисляется индекс оператора из алгебры СИО с матричными кусочно непрерьвньми коэффициентами в рефлексивном пространстве Орлича $L_{M}^{n}(\Gamma)$ на логарифмической кривой Карлесона $Г$.

2. Пространства Орлича и индексы Бойда. Основные сведения из теории пространств Орлича приведены, например, в [13]-[15].

Функиией Юнга назьвается выпуклая непрерывная функция $M: \mathbb{R}_{+} \rightarrow \mathbb{R}_{+}$, для которой $M(0)=0, M(x)>0$ при $x>0$,

$$
\lim _{x \rightarrow+0} \frac{M(x)}{x}=\lim _{x \rightarrow+\infty} \frac{x}{M(x)}=0 .
$$

Дополнительной к функции $M$ называется функция Юнга

$$
N(x):=\max _{y \geqslant 0}\{x y-M(y)\}
$$


Пусть Г- логарифмическая кривая Карлесона, ориентированная против часовой стрелки. Пространством Орлича $L_{M}(\Gamma)$ называется множество всех измеримых на Г комплекснозначных функций $u$, для каждой из которых при некотором $\lambda>0$ выполняется неравенство

$$
\int_{\Gamma} M\left(\frac{|u(\tau)|}{\lambda}\right)|d \tau|<\infty
$$

Пространство $L_{M}(\Gamma)$ снабдим нормой Орлича

$$
\|u\|_{M}:=\sup \left\{\int_{\Gamma}|u(\tau) v(\tau)||d \tau|: \int_{\Gamma} N(|v(\tau)|)|d \tau| \leqslant 1\right\} .
$$

Относительно этой нормы пространство $L_{M}(\Gamma)$ является банаховым.

В. Матушевска и В. Орлич [16] (см. также [15], [17]) связали с пространством $L_{M}(\Gamma)$ пару индексов. В общем случае перестановочно инвариантных пространств в работе Д. Бойда [18] были введены новые индексы, совпадающие с величинами, обратными к индексам Матушевской-Орлича в случае пространств $L_{M}(\Gamma)$. Нижний и верхний индексы Бойда $\alpha_{M}$ и $\beta_{M}$ определяются следующим образом:

$$
\alpha_{M}=\sup _{t>1} \Theta(t)=\lim _{t \rightarrow+\infty} \Theta(t), \quad \beta_{M}=\inf _{0<t<1} \Theta(t)=\lim _{t \rightarrow+0} \Theta(t), \quad \Theta(t)=-\frac{\log h(t)}{\log t},
$$

и для пространств Орлича [19]

$$
h(t)=\varlimsup_{x \rightarrow+\infty} \frac{M^{-1}(x)}{M^{-1}(t x)}, \quad t>0 .
$$

Индексы Бойда являются важными интерполящионными и геометрическими характеристиками перестановочно инвариантного пространства $L_{M}(\Gamma)$ (см. [14]-[19]). Например, пространство Орлича $L_{M}(\Gamma)$ рефлексивно тогда и только тогда, когда $0<\alpha_{M} \leqslant$ $\beta_{M}<1$.

Пространство Орлича, порожденное функцией Юнга $x^{p} / p, 1<p<\infty$, изоморфно пространству Лебега $L_{p}(\Gamma)$. Индексы Бойда пространства $L_{p}(\Gamma)$, где $1<p<\infty$, совпадают и равны $1 / p$. Примеры функций Юнга, порождающих рефлексивные пространства Орлича, не изоморфные пространствам Лебега, для которых индексы Бойда совпадают (различны) приведены, например, в [15, с. 93].

3. Критерий нётеровости. Линейньй ограниченньй оператор $A$ в банаховом пространстве $X$ назьвается нётеровым, если его образ замкнут в $X$ и конечны дефектные числа $n(A):=\operatorname{dim} \operatorname{Ker} A, d(A):=\operatorname{dim} \operatorname{Ker} A^{*}$. В этом случае индексом оператора $A$ назьвается число Ind $A:=n(A)-d(A)$.

Будем обозначать через $L_{M}^{n}(\Gamma)$ прямую сумму $n$ копий рефлексивного пространства Орлича $L_{M}(\Gamma)$. Через $\mathscr{L}:=\mathscr{L}\left(L_{M}^{n}(\Gamma)\right)$ обозначается банахова алгебра линейных ограниченных операторов в $L_{M}^{n}(\Gamma)$, а через $\mathscr{K}:=\mathscr{K}\left(L_{M}^{n}(\Gamma)\right)$ - идеал компактных операторов в $\mathscr{L}$.

Рассмотрим банахову алгебру $C(\Gamma)$ всех непрерывных функций на $\Gamma$, пусть $\Lambda(\Gamma)-$ множество функций, непрерывных в каждой точке контура $\Gamma$, кроме, быть может, конечного числа точек, в которых эти функции имеют конечные односторонние пределы. 
Замыкание множества $\Lambda(\Gamma)$ по норме $L_{\infty}(\Gamma)$ образует банахову алгебру $P C(\Gamma)$ всех кусочно-непрерьвных функций на $\Gamma$.

Пусть $C^{n \times n}(\Gamma)\left(\Lambda^{n \times n}(\Gamma), P C^{n \times n}(\Gamma), L_{\infty}^{n \times n}(\Gamma)\right)$ - множество $(n \times n)$-матрищ-функций, элементы которого принадлежат $C(\Gamma)\left(\Lambda(\Gamma), P C(\Gamma), L_{\infty}(\Gamma)\right.$ соответственно).

Пусть $I$ - тождественньй оператор, а оператор $S$ определен в пространстве $L_{M}^{n}(\Gamma)$ покомпонентно формулой (1). Рассмотрим наименьшую банахову подалгебру $\mathfrak{A}$ алгебры $\mathscr{L}$, содержащую операторы умножения на кусочно непрерывные матрицы-функции и оператор $S$. Сформулируем критерий нётеровости оператора из $\mathfrak{A}$ (см. [7]) в удобной для нас форме.

Пусть даны два действительных числа $a, b$, удовлетворяющие неравенству $0<a \leqslant$ $b<1$, два комплексных числа $z, w$ и действительное число $\delta$. Определим спиралевидный рожск [5]

$$
\mathscr{S}(z, w ; \delta ; a, b):=\{z, w\} \cup\left\{u \in \mathbb{C} \backslash\{z, w\}: \frac{1}{2 \pi}\left(\arg \frac{u-z}{u-w}-\delta \log \left|\frac{u-z}{u-w}\right|\right) \in[a, b]\right\} .
$$

Здесь значение $\arg (\cdot)$ выбирается так, чтобы $\arg (\cdot)-\delta \log |\cdot| \in[0,2 \pi)$. Легко видеть, что имеет место равенство

$$
\mathscr{S}(z, w ; \delta ; a, b)=\{z(1-\mu)+w \mu: \mu \in \mathscr{S}(0,1 ; \delta ; a, b)\} .
$$

Согласно [5] (двойную) логарифмическую спираль $\mathscr{S}(z, w ; \delta ; a, a)$ можно задать параметрически в виде

$$
\mathscr{S}(z, w ; \delta ; a, a):=\left\{\xi=\frac{w e^{\psi(1+i \delta)} e^{2 \pi i a}-z}{e^{\psi(1+i \delta)} e^{2 \pi i a}-1}: \psi \in[-\infty,+\infty]\right\} .
$$

Определим массивный иилиндр

$$
\mathfrak{N}:=\Gamma \times[0,1] \times\left[\alpha_{M}, \beta_{M}\right]
$$

Между пучком спиралевидных рожкков

$$
\mathfrak{S}:=\bigcup_{t \in \Gamma}\left(\{t\} \times \mathscr{S}\left(0,1 ; \delta(t) ; \alpha_{M}, \beta_{M}\right)\right)
$$

где $\delta(t)$ описывает степень закрученности кривой Г в точке $t($ см. (4)), и множеством $\mathfrak{N}$ можно установить биекцию по правилу

$$
\mathscr{F}: \mathfrak{N} \rightarrow \mathfrak{S}, \quad \mathscr{F}(t, x, \lambda)=(t, f(t, x, \lambda)),
$$

где

$$
f(t, x, \lambda)=\frac{e^{\varphi(t, x, \lambda)}}{e^{\varphi(t, x, \lambda)}-1}, \quad \varphi(t, x, \lambda)=(1+i \delta(t)) \log \frac{x}{1-x}+2 \pi i \lambda .
$$

Для $a \in P C^{n \times n}(\Gamma)$ введем матрицы-функции

$$
\begin{gathered}
U_{a}(t, x, \lambda):=a(t+0) f(t, x, \lambda)+a(t-0)(1-f(t, x, \lambda)), \\
V_{a}(t, x, \lambda):=a(t+0)(1-f(t, x, \lambda))+a(t-0) f(t, x, \lambda), \\
W_{a}(t, x, \lambda):=(a(t+0)-a(t-0)) \sqrt{f(t, x, \lambda)(1-f(t, x, \lambda)),}
\end{gathered}
$$

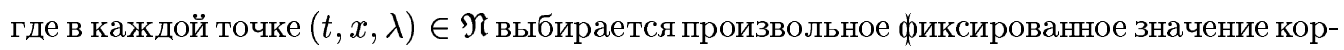
ня. 
Теорема 1. Справедливы следующие утверждения:

а) $\mathscr{K} \subset \mathfrak{A} \subset \mathscr{L}$ и факторалгебра $\mathfrak{A} / \mathscr{K}$ обратимо замкнута (наполнена) в алгебре Калкина $\mathscr{L} / \mathscr{K}$, т.е. если произвольный әлемент $A+\mathscr{K} \in \mathfrak{A} / \mathscr{K}$ обратим в алгебре $\mathscr{L} / \mathscr{K}$, то $(A+\mathscr{K})^{-1} \in \mathfrak{A} / \mathscr{K}$;

б) для каждой точки $(t, x, \lambda) \in \mathfrak{N}$ отображсние

$$
\omega_{t, x, \lambda}:\left\{a I: a \in P C^{n \times n}(\Gamma)\right\} \cup\{S\} \rightarrow \mathbb{C}^{2 n \times 2 n},
$$

определяемое равенствами

$$
\omega_{t, x, \lambda}(a I)=\left(\begin{array}{cc}
U_{a}(t, x, \lambda) & W_{a}(t, x, \lambda) \\
W_{a}(t, x, \lambda) & V_{a}(t, x, \lambda)
\end{array}\right), \quad \omega_{t, x, \lambda}(S)=\left(\begin{array}{cc}
E & O \\
O & -E
\end{array}\right)
$$

где О и $E$ - нулевая и единичная $(n \times n)$-матричь, расииряется до (непрерывного) гомоморфизма $\omega_{t, x, \lambda}: \mathfrak{A} \rightarrow \mathbb{C}^{2 n \times 2 n}$ банаховых алгебр, ядро которого содержит идеал $\mathscr{K}$;

в) оператор $A \in \mathfrak{A}$ нётеров в $L_{M}^{n}(\Gamma)$ тогда и только тогда, когда

$$
\operatorname{det} \omega_{t, x, \lambda}(A) \neq 0 \quad \text { для всех } \quad(t, x, \lambda) \in \mathfrak{N} .
$$

ДокаЗАТЕльСтво. Вложение $\mathscr{K} \subset \mathfrak{A}$ доказано в [7, лемма 9.1]. Так как (6) является биекцией $\mathfrak{N}$ на $\mathfrak{S}$, то

$$
\omega_{t, x, \lambda}(A)=\sigma_{t, \mu}(A+\mathscr{K}) \quad \text { для всех }(t, x, \lambda) \in \mathfrak{N},
$$

где $\mu=f(t, x, \lambda) \in \mathscr{S}\left(0,1 ; \delta(t) ; \alpha_{M}, \beta_{M}\right)$, а гомоморфизм $\sigma_{t, \mu}: \mathfrak{A} / \mathscr{K} \rightarrow \mathbb{C}^{2 n \times 2 n}$ для

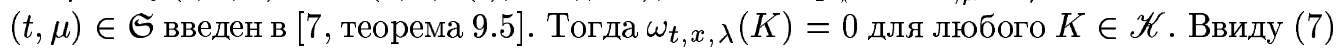
остальные утверждения теоремы 1 вытекают из теоремы 9.5 [7].

4. Символ и его детерминант. Матрицу-функцию $\mathscr{A}(t, x, \lambda)=\omega_{t, x, \lambda}(A)$ при $(t, x, \lambda) \in \mathfrak{N}$ будем называть символом оператора $A \in \mathfrak{A}$. Представим его в виде

$$
\mathscr{A}(t, x, \lambda)=\left(\begin{array}{ll}
\mathscr{A}_{11}(t, x, \lambda) & \mathscr{A}_{12}(t, x, \lambda) \\
\mathscr{A}_{21}(t, x, \lambda) & \mathscr{A}_{22}(t, x, \lambda)
\end{array}\right), \quad(t, x, \lambda) \in \mathfrak{N},
$$

где $\mathscr{A}_{i j}(t, x, \lambda)-(n \times n)$-матрицы-функции.

В пространстве $L_{M}^{n}(\Gamma)$ рассмотрим проекторы $P_{ \pm}:=(I \pm S) / 2$. Обозначим

$$
K:=\left(\max \left\{\left\|P_{+}\right\|_{\mathscr{L}},\left\|P_{-}\right\|_{\mathscr{L}}\right\}\right)^{2} .
$$

Семейство гомоморфизмов $\omega_{t, x, \lambda}$, вообще говоря, не является равномерно ограниченным по $(t, x, \lambda) \in \mathfrak{N}$. Однако, этим свойством обладают функции $\operatorname{det} \mathscr{A}, \operatorname{det} \mathscr{A}_{i i}(i=1,2)$. Более точно, если обозначить через

$$
|A|:=\inf _{T \in \mathscr{K}}\|A+T\|_{\mathscr{L}}
$$

существенную норму оператора $A \in \mathscr{L}$ (т.е. норму класса смежности $A+\mathscr{K}$ в алгебре Калкина $\mathscr{L} / \mathscr{K})$, а через

$$
\|a\|:=\sup _{(t, x, \lambda) \in \mathfrak{N}}|a(t, x, \lambda)|
$$

sup-норму функции $a: \mathfrak{N} \rightarrow \mathbb{C}$, то имеют место следующие теоремы. 
TeOpema 2. Ecлu $A \in \mathfrak{A}, m o$

$\|\operatorname{det} \mathscr{A}\| \leqslant(2 n) !(K|A|)^{2 n}, \quad\left\|\operatorname{det} \mathscr{A}_{i i}\right\| \leqslant n !(K|A|)^{n} \quad(i=1,2)$.

Теорема 3. Пусть последовательность операторов $A_{s} \in \mathfrak{A}$ равномерно сходится $\kappa$ оператору $A, u \mathscr{A}^{(s)}$ - последовательность символов операторов $A_{s}$. Тогда последовательность функиий $\operatorname{det} \mathscr{A}^{(s)}\left(\operatorname{det} \mathscr{A}_{11}^{(s)}, \operatorname{det} \mathscr{A}_{22}^{(s)}\right)$ равномерно сходится на множестве $\mathfrak{N} \kappa$ функиии $\operatorname{det} \mathscr{A}$ (det $\mathscr{A}_{11}$, $\operatorname{det} \mathscr{A}_{22}$ соответственно).

Для доказательства теорем 2 и 3 потребуется ряд вспомогательных утверждений. Обозначим через $v_{i}$ диагональную $(n \times n)$-матрицу, у которой элемент, стоящий на $i$-м месте равен 1 , а все остальные элементы равны 0 . Операторы

$$
\begin{aligned}
& Z_{1}(A ; i, j):=P_{+} v_{i} A v_{j} P_{+}, \quad Z_{2}(A ; i, j):=P_{+} v_{i} A v_{j} P_{-}, \\
& Z_{3}(A ; i, j):=P_{-} v_{i} A v_{j} P_{+}, \quad Z_{4}(A ; i, j):=P_{-} v_{i} A v_{j} P_{-},
\end{aligned}
$$

будем назьвать әлементарными операторами, ассоциированными с оператором $A$.

Лемма 1. Пусть операторы $A, B \in \mathfrak{A} u$

$$
A_{l}=Z_{k}(A ; i, j), \quad B_{l}=Z_{k}(B ; i, j), \quad 1 \leqslant l \leqslant m,
$$

где тройка $\{i, j, k\}$ зависит от $l u 1 \leqslant k \leqslant 4,1 \leqslant i, j \leqslant n$. Тогда

$$
\left|A_{1} A_{2} \cdots A_{m}-B_{1} B_{2} \cdots B_{m}\right| \leqslant K^{m}|A-B| \sum_{l=1}^{m}|B|^{l-1}|A|^{m-l} .
$$

ДокАЗАТЕЛЬСТво. По индукции доказьвается, что

$$
\left|A_{1} A_{2} \cdots A_{m}-B_{1} B_{2} \cdots B_{m}\right| \leqslant \sum_{l=1}^{m}\left(\prod_{j=1}^{l-1}\left|B_{j}\right| \cdot\left|A_{l}-B_{l}\right| \cdot \prod_{j=l+1}^{m}\left|A_{j}\right|\right) .
$$

Пусть $1 \leqslant l \leqslant m$. Тогда с учетом определения постоянной $K$

$$
\left|A_{l}-B_{l}\right|=\left|Z_{k}(A ; i, j)-Z_{k}(B ; i, j)\right|=\left|Z_{k}(A-B ; i, j)\right| \leqslant K|A-B| .
$$

Следовательно, $\left|A_{l}\right| \leqslant K|A|,\left|B_{l}\right| \leqslant K|B|$. Отсюда и из неравенств (9), (10) вытекает неравенство (8).

Через $J(a ; i, j)$ обозначим $(2 n \times 2 n)$-матрицу, у которой элемент, стоящий в $i$-й строке и $j$-м столбце, равен $a$, а все остальные элементы равны 0. Представим символ $\mathscr{A}$ произвольного оператора $A \in \mathfrak{A}$ в виде

$$
\mathscr{A}(t, x, \lambda)=\left(a_{i j}(t, x, \lambda)\right)_{i, j=1}^{2 n}, \quad(t, x, \lambda) \in \mathfrak{N} .
$$

Вычислим символы элементарных операторов $(1 \leqslant i, j \leqslant n)$ :

$$
\begin{aligned}
\left(\mathscr{Z}_{1}(A ; i, j)\right)(t, x, \lambda) & =J(1 ; i, i) \mathscr{A}(t, x, \lambda) J(1 ; j, j)=J\left(a_{i j}(t, x, \lambda) ; i, j\right), \\
\left(\mathscr{Z}_{2}(A ; i, j)\right)(t, x, \lambda) & =J(1 ; i, i) \mathscr{A}(t, x, \lambda) J(1 ; j+n, j+n) \\
& =J\left(a_{i, j+n}(t, x, \lambda) ; i, j+n\right), \\
\left(\mathscr{Z}_{3}(A ; i, j)\right)(t, x, \lambda) & =J(1 ; i+n, i+n) \mathscr{A}(t, x, \lambda) J(1 ; j, j) \\
& =J\left(a_{i+n, j}(t, x, \lambda) ; i+n, j\right), \\
\left(\mathscr{Z}_{4}(A ; i, j)\right)(t, x, \lambda) & =J(1 ; i+n, i+n) \mathscr{A}(t, x, \lambda) J(1 ; j+n, j+n) \\
& =J\left(a_{i+n, j+n}(t, x, \lambda) ; i+n, j+n\right) .
\end{aligned}
$$

Таким образом, матрицы вида $J\left(a_{i j}(t, x, \lambda) ; i, j\right)$, где $a_{i j}(t, x, \lambda)$ есть $(i, j)$-й элемент символа $\mathscr{A}(t, x, \lambda)$, также являются символами операторов из алгебры $\mathfrak{A}$. 
Лемма 2. Пусть $A$ - оператор из алгебры $\mathfrak{A}$, символ которого имеет вид

$$
\mathscr{A}(t, x, \lambda)=J(a(t, x, \lambda) ; i, i), \quad(t, x, \lambda) \in \mathfrak{N}, \quad 1 \leqslant i \leqslant 2 n .
$$

Тогда $\|a\| \leqslant|A|$.

ДокАЗАТЕЛьСтво. Идея доказательства заимствована из [2, теорема 4.1]. Допустим, что существует точка $\left(t_{0}, x_{0}, \lambda_{0}\right) \in \mathfrak{N}$, для которой $|A|<\left|a\left(t_{0}, x_{0}, \lambda_{0}\right)\right|$. В этом случае $|A| /\left|a\left(t_{0}, x_{0}, \lambda_{0}\right)\right|<1$. Тогда оператор $B=I-\left(a\left(t_{0}, x_{0}, \lambda_{0}\right)\right)^{-1} A$ нётеров, а его символ - диагональная матрица

$$
\mathscr{B}(t, x, \lambda)=\operatorname{diag}\left\{1, \ldots, 1,1-\frac{a(t, x, \lambda)}{a\left(t_{0}, x_{0}, \lambda_{0}\right)}, 1, \ldots, 1\right\}
$$

где на $i$-м месте стоит элемент $1-a(t, x, \lambda) / a\left(t_{0}, x_{0}, \lambda_{0}\right)$. Следовательно,

$$
\operatorname{det} \mathscr{B}\left(t_{0}, x_{0}, \lambda_{0}\right)=1-\frac{a\left(t_{0}, x_{0}, \lambda_{0}\right)}{a\left(t_{0}, x_{0}, \lambda_{0}\right)}=0,
$$

что противоречит утверждению в) теоремы 1.

Лемма 3. Если $A, B \in \mathfrak{A}, m o\left\|a_{i i}-b_{i i}\right\| \leqslant K|A-B|$ для всех $i, 1 \leqslant i \leqslant 2 n$.

ДокАЗАТЕльСтво. Если $1 \leqslant i \leqslant n$, то из леммы 2 и равенств $(11),(14)$ вытекает, что

$$
\left\|a_{i i}-b_{i i}\right\| \leqslant\left|Z_{1}(A-B ; i, i)\right|, \quad\left\|a_{i+n, i+n}-b_{i+n, i+n}\right\| \leqslant\left|Z_{4}(A-B ; i, i)\right| .
$$

Применив к правым частям последних двух неравенств лемму 1 при $m=1$, получим требуемое утверждение.

Обозначим через $\mathfrak{S}_{2 n}$ симметрическую группу перестановок степени $2 n$. Пусть даны различные натуральные числа $1 \leqslant p_{1}<\cdots<p_{m} \leqslant 2 n$. Lиклом $\left(p_{1}, \ldots, p_{m}\right) \in \mathfrak{S}_{2 n}$ назьвается перестановка, действующая по правилу: элементы, отличные от $p_{i}, 1 \leqslant i \leqslant m$, переходят в себя; $p_{i}$ переходит в $p_{i+1}, 1 \leqslant i \leqslant m-1$, а $p_{m}$ переходит в $p_{1}$. Число $m$ называется длиной иикла. Обозначим

$$
C_{A,\left(p_{1}, \ldots, p_{m}\right)}(t, x, \lambda):=a_{p_{1} p_{2}}(t, x, \lambda) \cdots a_{p_{m-1} p_{m}}(t, x, \lambda) \cdot a_{p_{m} p_{1}}(t, x, \lambda) .
$$

Лемма 4. Если $A, B \in \mathfrak{A} u\left(p_{1}, \ldots, p_{m}\right) \in \mathfrak{S}_{2 n}-$ иикл длины $m$, то

$$
\left\|C_{A,\left(p_{1}, \ldots, p_{m}\right)}-C_{B,\left(p_{1}, \ldots, p_{m}\right)}\right\| \leqslant K^{m}|A-B| \sum_{l=1}^{m}|B|^{l-1}|A|^{m-l} .
$$


ДоКАЗАТЕЛЬСТво. Непосредственной проверкой можно убедиться в том, что

$$
\begin{aligned}
& J\left(a_{p_{1} p_{2}}(t, x, \lambda) ; p_{1}, p_{2}\right) \cdots J\left(a_{p_{m-1} p_{m}}(t, x, \lambda) ; p_{m-1}, p_{m}\right) \cdot J\left(a_{p_{m} p_{1}}(t, x, \lambda) ; p_{m}, p_{1}\right) \\
& \quad=J\left(C_{A,\left(p_{1}, \ldots, p_{m}\right)}(t, x, \lambda) ; p_{1}, p_{1}\right) \\
& J\left(b_{p_{1} p_{2}}(t, x, \lambda) ; p_{1}, p_{2}\right) \cdots J\left(b_{p_{m-1} p_{m}}(t, x, \lambda) ; p_{m-1}, p_{m}\right) \cdot J\left(b_{p_{m} p_{1}}(t, x, \lambda) ; p_{m}, p_{1}\right) \\
& \quad=J\left(C_{B,\left(p_{1}, \ldots, p_{m}\right)}(t, x, \lambda) ; p_{1}, p_{1}\right) .
\end{aligned}
$$

Каждая матрица, стоящая в левой части равенства (15) (соответственно (16)), является символом элементарного оператора $A_{l}, 1 \leqslant l \leqslant m$ (соответственно $B_{l}$ ), ассоциированного с оператором $A$ (соответственно $B$ ) по формулам (11)-(14). В итоге, из равенств (15), (16) вытекает, что

$$
\begin{aligned}
& J\left(C_{A,\left(p_{1}, \ldots, p_{m}\right)}(t, x, \lambda)-C_{B,\left(p_{1}, \ldots, p_{m}\right)}(t, x, \lambda) ; p_{1}, p_{1}\right) \\
& \quad=\omega_{t, x, \lambda}\left(A_{1} A_{2} \cdots A_{m}-B_{1} B_{2} \cdots B_{m}\right) .
\end{aligned}
$$

По лемме 2

$$
\left\|C_{A,\left(p_{1}, \ldots, p_{m}\right)}-C_{B,\left(p_{1}, \ldots, p_{m}\right)}\right\| \leqslant\left|A_{1} A_{2} \cdots A_{m}-B_{1} B_{2} \cdots B_{m}\right| .
$$

Применив к правой части последнего неравенства лемму 1 , получим требуемое утверждение.

ДоКАЗАТЕЛЬСТВО ТЕОРЕМЫ 3. Из выражения определителя через элементы матрицы вытекает, что

$$
\left\|\operatorname{det} \mathscr{A}-\operatorname{det} \mathscr{A}^{(s)}\right\| \leqslant \sum_{\pi \in \mathfrak{S}_{2 n}}\left\|\prod_{j=1}^{2 n} a_{j i_{j}}-\prod_{j=1}^{2 n} a_{j i_{j}}^{(s)}\right\|,
$$

где $i_{j}=\pi(j)$. Перестановку $\pi \in \mathfrak{S}_{2 n}$, отличную от тождественной, разложим в произведение независимых циклов:

$$
\pi=\left(\begin{array}{cccc}
1 & 2 & \ldots & 2 n \\
i_{1} & i_{2} & \ldots & i_{2 n}
\end{array}\right)=\left(p_{1}^{(1)}, \ldots, p_{m(1)}^{(1)}\right) \cdots\left(p_{1}^{(r)}, \ldots, p_{m(r)}^{(r)}\right)
$$

где $r$ - число циклов, $m(l)$ - длина $l$-го цикла. Обозначим через $k$ число элементов, остаюшихся на месте под действием перестановки $\pi$. Очевидно,

$$
k=2 n-\sum_{l=1}^{r} m(l) .
$$

Для тождественной перестановки $r=0, k=2 n$. Рассмотрим множество

$$
\left\{q_{1}, \ldots, q_{k}\right\}:=\{1, \ldots, 2 n\} \backslash \bigcup_{l=1}^{r}\left\{p_{1}^{(l)}, \ldots, p_{m(l)}^{(l)}\right\}
$$

Обозначим

$$
C_{l}(t, x, \lambda):=C_{A,\left(p_{1}^{(l)}, \ldots, p_{m(l)}^{(l)}\right)}(t, x, \lambda), \quad C_{l}^{(s)}(t, x, \lambda):=C_{A_{s},\left(p_{1}^{(l)}, \ldots, p_{m(l)}^{(l)}\right)}(t, x, \lambda) .
$$


Аналогично (9) оценим каждое слагаемое, стоящее в формуле (17) справа:

$$
\begin{aligned}
\left\|\prod_{j=1}^{2 n} a_{j i_{j}}-\prod_{j=1}^{2 n} a_{j i_{j}}^{(s)}\right\| & =\left\|\prod_{l=1}^{r} C_{l} \cdot \prod_{l=1}^{k} a_{q_{l} q_{l}}-\prod_{l=1}^{r} C_{l}^{(s)} \cdot \prod_{l=1}^{k} a_{q_{l} q_{l}}^{(s)}\right\| \\
\leqslant & \prod_{l=1}^{r}\left\|C_{l}^{(s)}\right\| \cdot \sum_{l=1}^{k}\left(\prod_{j=1}^{l-1}\left\|a_{q_{j} q_{j}}^{(s)}\right\| \cdot\left\|a_{q_{l} q_{l}}-a_{q_{l} q_{l}}^{(s)}\right\| \cdot \prod_{j=l+1}^{k}\left\|a_{q_{j} q_{j}}\right\|\right) \\
& +\prod_{l=1}^{k}\left\|a_{q_{l} q_{l}}\right\| \cdot \sum_{l=1}^{r}\left(\prod_{j=1}^{l-1}\left\|C_{j}^{(s)}\right\| \cdot\left\|C_{l}-C_{l}^{(s)}\right\| \cdot \prod_{j=l+1}^{r}\left\|C_{j}\right\|\right) .
\end{aligned}
$$

Так как последовательность $A_{s}$ равномерно сходится к $A$, то $\left|A_{s}\right| \leqslant 2|A|$ начиная с некоторого номера $s_{0}$. Тогда для всех $s \geqslant s_{0}$ из леммы 3 при $1 \leqslant j \leqslant k$ следует, что

$$
\left\|a_{q_{j} q_{j}}-a_{q_{j} q_{j}}^{(s)}\right\| \leqslant K\left|A-A_{s}\right|, \quad\left\|a_{q_{j} q_{j}}\right\| \leqslant K|A|, \quad\left\|a_{q_{j} q_{j}}^{(s)}\right\| \leqslant 2 K|A| .
$$

Из леммы 4 для всех $1 \leqslant l \leqslant r$ вытекает оценка

$$
\left\|C_{l}-C_{l}^{(s)}\right\| \leqslant K^{m(l)}\left|A-A_{s}\right| \sum_{j=1}^{m(l)}\left|A_{s}\right|^{j-1}|A|^{m(l)-j} .
$$

Тогда для всех $s \geqslant s_{0}$ и всех $1 \leqslant l \leqslant r$

$$
\begin{gathered}
\left\|C_{l}\right\| \leqslant(K|A|)^{m(l)}, \quad\left\|C_{l}^{(s)}\right\| \leqslant\left(K\left|A_{s}\right|\right)^{m(l)} \leqslant(2 K|A|)^{m(l)} \\
\left\|C_{l}-C_{l}^{(s)}\right\| \leqslant\left(2^{m(l)}-1\right) K^{m(l)}|A|^{m(l)-1}\left|A-A_{s}\right|
\end{gathered}
$$

В итоге из неравенств (20)-(23) с учетом равенства (19) вытекает, что

$$
\begin{aligned}
& \left\|\prod_{j=1}^{2 n} a_{j i_{j}}-\prod_{j=1}^{2 n} a_{j i_{j}}^{(s)}\right\| \\
& \quad \leqslant\left(2^{2 n-k} \sum_{l=1}^{k} 2^{l-1}+\sum_{l=1}^{r}\left(\prod_{j=1}^{l-1} 2^{m(j)} \cdot\left(2^{m(l)}-1\right)\right)\right) K^{2 n}|A|^{2 n-1}\left|A-A_{s}\right| \\
& \quad=\left(2^{2 n}-1\right) K^{2 n}|A|^{2 n-1}\left|A-A_{s}\right| .
\end{aligned}
$$

Из неравенств (17) и (24) получаем

$$
\left\|\operatorname{det} \mathscr{A}-\operatorname{det} \mathscr{A}^{(s)}\right\| \leqslant(2 n) !\left(2^{2 n}-1\right) K^{2 n}|A|^{2 n-1}\left|A-A_{s}\right| .
$$

Осталось показать, что последовательности функций $\operatorname{det} \mathscr{A}_{i i}^{(s)}(i=1,2)$ равномерно сходятся к функциям $\operatorname{det} \mathscr{A}_{i i}$ соответственно. Пусть, например, $i=2$. Рассмотрим последовательность операторов $B_{s}:=P_{+}+P_{-} A_{s} P_{-}$, равномерно сходящуюся к оператору $B:=P_{+}+P_{-} A P_{-}$. Так как $\operatorname{det} \mathscr{B}=\operatorname{det} \mathscr{A}_{22}, \operatorname{det} \mathscr{B}^{(s)}=\operatorname{det} \mathscr{A}_{22}^{(s)}$, равномерная сходимость $\operatorname{det} \mathscr{A}_{22}^{(s)} \rightarrow \operatorname{det} \mathscr{A}_{22}$ вытекает из (25). 
ДоКАЗАТЕЛЬСТВо ТЕОРЕМЫ 2. Имеем

$$
\|\operatorname{det} \mathscr{A}\| \leqslant \sum_{\pi \in \mathfrak{S}_{2 n}}\left\|\prod_{j=1}^{2 n} a_{j i_{j}}\right\|
$$

Если перестановка $\pi$ разлагается в произведение (18), то согласно доказательству теоремы 3

$$
\left\|\prod_{j=1}^{2 n} a_{j i_{j}}\right\| \leqslant \prod_{l=1}^{r}\left\|C_{l}\right\| \cdot \prod_{j=1}^{k}\left\|a_{q_{j} q_{j}}\right\|, \quad\left\|C_{l}\right\| \leqslant(K|A|)^{m(l)}, \quad\left\|a_{q_{j} q_{j}}\right\| \leqslant K|A| .
$$

Отсюда ввиду (26) и (19)

$$
\|\operatorname{det} \mathscr{A}\| \leqslant \sum_{\pi \in \mathfrak{S}_{2 n}}(K|A|)^{2 n}=(2 n) !(K|A|)^{2 n} .
$$

Неравенство для блоков $\mathscr{A}_{i i}(i=1,2)$ доказывается аналогично.

5. Формула индекса. Рассмотрим цилиндр $\mathfrak{M}=\Gamma \times[0,1]$. Согласно [1], [2] введем на $\mathfrak{M}$ топологию следующим образом. Определим окрестности

$$
\begin{aligned}
\Omega(t, 0) & :=\{(\tau, x) \in \mathfrak{M}:|\tau-t|<\delta, \tau \prec t, x \in[0,1]\} \cup\{(t, x) \in \mathfrak{M}: x \in[0, \varepsilon)\}, \\
\Omega(t, 1) & :=\{(\tau, x) \in \mathfrak{M}:|\tau-t|<\delta, t \prec \tau, x \in[0,1]\} \cup\{(t, x) \in \mathfrak{M}: x \in(\varepsilon, 1]\}, \\
\Omega\left(t, x_{0}\right) & :=\left\{(t, x) \in \mathfrak{M}: x \in\left(x_{0}-\delta_{1}, x_{0}+\delta_{2}\right)\right\}
\end{aligned}
$$

где $x_{0} \neq 0,1,0<\delta_{1}<x_{0}, 0<\delta_{2}<1-x_{0}, 0<\varepsilon<1$. Рассмотрим функцию

$$
F(t, x, \lambda):=\prod_{j=1}^{k} U_{a_{j}}(t, x, \lambda), \quad(t, x, \lambda) \in \mathfrak{N}
$$

где $a_{j} \in \Lambda(\Gamma), 1 \leqslant j \leqslant k, k \geqslant 1$. Допустим, что $F(t, x, \lambda) \neq 0$ для всех $(t, x, \lambda) \in \mathfrak{N}$. Зафиксируем $\lambda \in\left[\alpha_{M}, \beta_{M}\right]$. Несложно видеть, что функция $F(\cdot, \cdot, \lambda)$ непрерьвна на цилиндре $\mathfrak{M}$. Образ этой функции - непрерьвная замкнутая кривая, не проходящая через 0, которую можно естественным образом ориентировать: в точках непрерывности функций $a_{j}$ движение по кривой определяется изменением $t$ по контуру $\Gamma$ в положительном направлении, а вдоль дополнительных спиралей, соединяющих точки разрьва функций $a_{j}$, движение определяется изменением $x$ от 0 до 1 . Определим индекс функции $F$ как число оборотов описанной выше кривой вокруг начала координат. Так как семейство функций $F(\cdot, \cdot, \lambda)$ непрерьвно по $\lambda$, то введенный индекс не зависит от $\lambda \in\left[\alpha_{M}, \beta_{M}\right]$. Будем обозначать его через $\operatorname{ind}_{\mathfrak{N}} F$.

Обозначим через $\mathfrak{F}(\mathfrak{N})$ класс функций $H: \mathfrak{N} \rightarrow \mathbb{C}$, удовлетворяющих следующим условиям:

i) $H(t, x, \lambda) \neq 0$ для всех $(t, x, \lambda) \in \mathfrak{N}$;

ii) функция $H$ представима в виде равномерного предела по $(t, x, \lambda) \in \mathfrak{N}$ последовательности функций $F_{s}$ вида $(27)$. 
Так как цилиндр $\mathfrak{M}$ является компактом, в случае выполнения условия іi) функция $H(\cdot, \cdot, \lambda)$ непрерьвна на цилиндре $\mathfrak{M}$ при любом $\lambda \in\left[\alpha_{M}, \beta_{M}\right]$. Начиная с некоторого номера $s_{0}$, числа ind ${ }_{\mathfrak{N}} F_{s}$ не зависят от $s$. Индексом функции $H \in \mathfrak{F}(\mathfrak{N})$ назовем

$$
\operatorname{ind}_{\mathfrak{N}} H:=\lim _{s \rightarrow \infty} \operatorname{ind}_{\mathfrak{N}} F_{s}
$$

Легко видеть, что так определенный индекс не зависит от выбора последовательности функций $F_{s}$ вида (27). Отметим, что введенный индекс обладает естественным логарифмическим свойством: если $F, H \in \mathfrak{F}(\mathfrak{N})$, то $F H \in \mathfrak{F}(\mathfrak{N})$, при этом

$$
\operatorname{ind}_{\mathfrak{N}} F H=\operatorname{ind}_{\mathfrak{N}} F+\operatorname{ind}_{\mathfrak{N}} H \text {. }
$$

Для доказательства формулы индекса нам потребуются некоторые вспомогательные факты.

Лемма 5. Пусть $a, b \in L_{\infty}^{n \times n}(\Gamma)$. Если оператор $a P_{+}+b P_{-}$нётеров в $L_{M}^{n}(\Gamma)$, mo

$$
\underset{t \in \Gamma}{\operatorname{essinf}}|\operatorname{det} a(t)|>0, \quad \underset{t \in \Gamma}{\operatorname{essinf}}|\operatorname{det} b(t)|>0 .
$$

Это утверждение доказывается по аналогии с леммой 2 из [20].

Непрерывная матрица-функция на кривой Г называется неособенной, если она невырождена в каждой точке $t \in \Gamma$. Пусть $a \in L_{\infty}^{n \times n}(\Gamma)$. Обозначим $R_{a}:=a P_{+}+P_{-}$.

Лемма 6. Пусть $G \in C^{n \times n}(\Gamma)$. Если оператор $R_{G}$ нётеров, то

$$
\text { Ind } R_{G}=-\frac{1}{2 \pi}\{\operatorname{Arg} \operatorname{det} G(t)\}_{\Gamma}
$$

ДокАЗАтЕльство. Если оператор $R_{G}$ нётеров, то по лемме $5 \operatorname{det} G(t) \neq 0$ для всех $t \in \Gamma$. Значит, матрища-функция $G(t)$ гомотопна матрице-функции $\widetilde{G}(t)=\operatorname{diag}\left\{t^{\varkappa}, 1\right.$, $\ldots, 1\}$, где $\varkappa=\frac{1}{2 \pi}\{\mathrm{Arg} \operatorname{det} G(t)\}_{\Gamma}$ - индекс Коши (см. добавление VI Б. Боярского в [21, c. 484-486]). По теореме об устойчивости индекса нётерова оператора $\operatorname{Ind} R_{G}=\operatorname{Ind} R_{\tilde{G}}$. Очевидно, что Ind $R_{\widetilde{G}}=\operatorname{Ind}\left(t^{\varkappa} P_{+}+P_{-}\right)=-\varkappa$.

Tеорема 4. Пусть $G \in P C(\Gamma)$. Оператор $R_{G}$ нётеров в рефлексивном пространстве Орлича $L_{M}(\Gamma)$ тогда и только тогда, когда $U_{G}(t, x, \lambda) \neq 0$ для всех $(t, x, \lambda) \in \mathfrak{N} . \quad$ Eсли оператор $R_{G}$ нётеров, то функиия $U_{G} \in \mathfrak{F}(\mathfrak{N})$, Ind $R_{G}=$ $-\operatorname{ind}_{\mathfrak{N}} U_{G}$.

Теорема 4 вытекает из [7, теорема 8.7] и равенства (5).

Teорема 5. Пусть $G \in \Lambda^{n \times n}(\Gamma)$. Ecли оператор $R_{G}$ нётеров в $L_{M}^{n}(\Gamma)$, то функиия $\operatorname{det} U_{G}$ представима в виде (27) и принадлежит классу $\mathfrak{F}(\mathfrak{N})$, Ind $R_{G}=$ $-\operatorname{ind}_{\mathfrak{N}} \operatorname{det} U_{G}$.

ДокАЗАтЕльство. Так как оператор $R_{G}$ нётеров, согласно утверждению в) теоремы 1 его символ

$$
\mathscr{R}_{G}(t, x, \lambda)=\left(\begin{array}{cc}
U_{G}(t, x, \lambda) & O \\
W_{G}(t, x, \lambda) & E
\end{array}\right)
$$

обратим, т.е. $\operatorname{det} \mathscr{R}_{G}(t, x, \lambda)=\operatorname{det} U_{G}(t, x, \lambda) \neq 0$ для всех $(t, x, \lambda) \in \mathfrak{N}$. 
По лемме $5 \operatorname{det} G(t \pm 0) \neq 0$ для всех $t \in \Gamma$. Тогда по лемме 2 из [22] матрица-функция $G$ представима в виде $G(t)=C_{1}(t) Y(t) C_{2}(t)$, где $C_{i}, i=1,2,-$ непрерьвные неособенные матрицы-функции, $Y \in \Lambda^{n \times n}(\Gamma)$ - верхнетреугольная матрица-функция с диагональными элементами $y_{j}, 1 \leqslant j \leqslant n$. Легко видеть, что в этом случае

$$
\operatorname{det} U_{G}(t, x, \lambda)=\operatorname{det} C_{1}(t) \cdot \prod_{j=1}^{n} U_{y_{j}}(t, x, \lambda) \cdot \operatorname{det} C_{2}(t) .
$$

Так как $C_{i} \in C^{n \times n}(\Gamma), i=1,2$, очевидно,

$$
\operatorname{det} C_{i}(t)=U_{\operatorname{det} C_{i}}(t, x, \lambda), \quad(t, x, \lambda) \in \mathfrak{N} .
$$

Потому функция $\operatorname{det} U_{G}$ имеет вид $(27)$ и принадлежит классу $\mathfrak{F}(\mathfrak{N})$. Для непрерьвных на $Г$ функций введенный в начале пункта индекс совпадает с индексом Коши:

$$
\operatorname{ind}_{\mathfrak{N}} \operatorname{det} C_{i}=\frac{1}{2 \pi}\left\{\operatorname{Arg} \operatorname{det} C_{i}(t)\right\}_{\Gamma}, \quad i=1,2 .
$$

Так как $\operatorname{det} U_{G}(t, x, \lambda) \neq 0$ для всех $(t, x, \lambda) \in \mathfrak{N}$, из равенства (28) вытекает, что $U_{y_{j}}(t, x, \lambda) \neq 0$ для всех $1 \leqslant j \leqslant n$ и $(t, x, \lambda) \in \mathfrak{N}$. По теореме 4 операторы $R_{y_{j}}$ нётеровы,

$$
\text { Ind } R_{y_{j}}=-\operatorname{ind}_{\mathfrak{N}} U_{y_{j}}, \quad 1 \leqslant j \leqslant n .
$$

Из результатов о нётеровости блочных операторов (см., например, [23]) следует, что оператор $R_{Y}$ нётеров и его индекс вычисляется по формуле

$$
\text { Ind } R_{Y}=\sum_{j=1}^{n} \operatorname{Ind} R_{y_{j}}
$$

Несложно доказать, что

$$
\text { Ind } R_{G}=\text { Ind } R_{C_{1}}+\text { Ind } R_{Y}+\text { Ind } R_{C_{2}} .
$$

Действительно, согласно утверждению в) теоремы 1 операторы $R_{C_{i}}, i=1,2$, нётеровы. Так как $R_{G}=\left(P_{+} G P_{+}+P_{-}\right)\left(I+P_{-} G P_{+}\right)$, где оператор $I+P_{-} G P_{+}$обратим: $\left(I+P_{-} G P_{+}\right)^{-1}=I-P_{-} G P_{+}$, оператор $R_{G}$ нётеров тогда и только тогда, когда оператор $P_{+} G P_{+}+P_{-}$нётеров, причем

$$
\text { Ind } R_{G}=\operatorname{Ind}\left(P_{+} G P_{+}+P_{-}\right) \text {. }
$$

Из $\left[7\right.$, лемма 5.6] следует, что если $a \in C^{n \times n}(\Gamma)$, то операторы $a P_{ \pm}-P_{ \pm} a I$ компактны; поэтому справедливо равенство

$$
\left(P_{+} C_{1} P_{+}+P_{-}\right)\left(P_{+} Y P_{+}+P_{-}\right)\left(P_{+} C_{2} P_{+}+P_{-}\right)=P_{+} G P_{+}+P_{-}+K,
$$

где $K \in \mathscr{K}$. Отсюда и из (33) вытекает (32).

Из леммы 6 и равенств (28)-(32) следует, что

$$
\begin{aligned}
\text { Ind } R_{G} & =\operatorname{Ind} R_{C_{1}}+\sum_{j=1}^{n} \operatorname{Ind} R_{y_{j}}+\operatorname{Ind} R_{C_{2}} \\
& =-\left(\operatorname{ind}_{\mathfrak{N}} \operatorname{det} C_{1}+\sum_{j=1}^{n} \operatorname{ind}_{\mathfrak{N}} U_{y_{j}}+\operatorname{ind} \mathfrak{N}_{\mathfrak{N}} \operatorname{det} C_{2}\right)=-\operatorname{ind} \mathfrak{N}_{\mathfrak{N}} \operatorname{det} U_{G} .
\end{aligned}
$$


Teорема 6. Пусть $A \in \mathfrak{A}$ нётеров в $L_{M}^{n}(\Gamma)$. Тогда функиия

$$
Q(t, x, \lambda)=\frac{\operatorname{det} \mathscr{A}(t, x, \lambda)}{\operatorname{det} \mathscr{A}_{22}(t, 0, \lambda) \operatorname{det} \mathscr{A}_{22}(t, 1, \lambda)}
$$

принадлежит классу $\mathfrak{F}(\mathfrak{N})$,

$$
\text { Ind } A=-\operatorname{ind}_{\mathfrak{N}} Q \text {. }
$$

ДокАЗАТЕльСтво. Доказательство проведем в три этапа.

1) Пусть $A=a P_{+}+b P_{-}$, где $a, b \in \Lambda^{n \times n}(\Gamma)$. Если оператор $A$ нётеров в $L_{M}^{n}(\Gamma)$, то по лемме $5 \operatorname{det} b(t \pm 0) \neq 0$ для всех $t \in \Gamma$. Тогда оператор $R_{b^{-1}}$ нёетеров, Ind $A=\operatorname{Ind} R_{b^{-1}} a$. По аналогии с леммой 1.1 из [1] или леммой 2.1 из [2] доказьвается, что

$$
\operatorname{det} \mathscr{A}(t, x, \lambda)=\operatorname{det} U_{b^{-1} a}(t, x, \lambda) \operatorname{det} \mathscr{A}_{22}(t, 0, \lambda) \operatorname{det} \mathscr{A}_{22}(t, 1, \lambda) .
$$

Отсюда и из теоремы 5 следует, что функция $Q=\operatorname{det} U_{b^{-1} a}$ имеет вид $(27)$ и принадлежит классу $\mathfrak{F}(\mathfrak{N})$,

$$
\text { Ind } R_{b^{-1} a}=-\operatorname{ind}_{\mathfrak{N}} \operatorname{det} U_{b^{-1} a}=-\operatorname{ind}_{\mathfrak{N}} Q \text {. }
$$

2) Пусть

$$
A=\sum_{j=1}^{k} A_{j 1} A_{j 2} \cdots A_{j r},
$$

где $A_{j l}=a_{j l} P_{+}+b_{j l} P_{-}, a_{j l}, b_{j l} \in \Lambda^{n \times n}(\Gamma), 1 \leqslant l \leqslant r, k \geqslant 1$, и оператор $A$ нётеров. Этот случай сводится к предыдущему с помощю процедуры линейного растяжения точно так же, как и в $[1$, теорема 7.1$]$ или в [2, теорема 3.1$]$. Таким образом, в этом случае функция $Q$ представима в виде $(27)$ и принадлежит $\mathfrak{F}(\mathfrak{N})$, при этом справедлива формула индекса (34).

3) Пусть $A$ - произвольный нётеров оператор из алгебры $\mathfrak{A}$. Тогда согласно утверждению в) теоремы $1 \operatorname{det} \mathscr{A}(t, x, \lambda) \neq 0$ для любого $(t, x, \lambda) \in \mathfrak{N}$. По теореме 2 функция $\operatorname{det} \mathscr{A}_{22}(t, x, \lambda)$ равномерно ограничена по $(t, x, \lambda) \in \mathfrak{N}$. Следовательно, $Q(t, x, \lambda) \neq 0$ для всех $(t, x, \lambda) \in \mathfrak{N}$. Оператор $A$ является равномерным пределом нётеровых операторов $A_{s}$ вида $(35)$ с символами $\mathscr{A}^{(s)}$. Согласно теореме 3 последовательности $\operatorname{det} \mathscr{A}^{(s)}(t$,

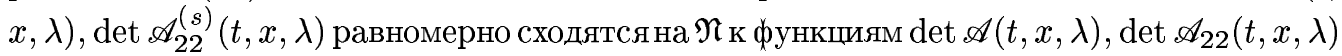
соответственно. Тогда последовательность функций

$$
Q_{s}(t, x, \lambda)=\frac{\operatorname{det} \mathscr{A}^{(s)}(t, x, \lambda)}{\operatorname{det} \mathscr{A}_{22}^{(s)}(t, 0, \lambda) \operatorname{det} \mathscr{A}_{22}^{(s)}(t, 1, \lambda)}
$$

равномерно сходится к функции $Q(t, x, \lambda)$ на множестве $\mathfrak{N}$. Как было отмечено выше в этапе 2$)$, функции $Q_{s}$ представимы в виде $(27)$. Тогда $Q \in \mathfrak{F}(\mathfrak{N})$ и по определению индекса функции из класса $\mathfrak{F}(\mathfrak{N})$

$$
\operatorname{ind}_{\mathfrak{N}} Q=\lim _{s \rightarrow \infty} \operatorname{ind}_{\mathfrak{N}} Q_{s} .
$$

Окончательно,

$$
\text { Ind } A=\lim _{s \rightarrow \infty} \operatorname{Ind} A_{s}=-\lim _{s \rightarrow \infty} \operatorname{ind}_{\mathfrak{N}} Q_{s}=-\operatorname{ind}_{\mathfrak{N}} Q .
$$




\section{СПИСОК ЦИТИРОВАННОЙ ЛИТЕРАТУРЫ}

[1] Гохберг И. Ц., Крупник Н. Я. Об алгебре, порожденной одномерными сингулярными интегральными операторами с кусочно непрерьвньпи коэффициентами // Функцион. анализ и его прилож. 1970. Т. 4. №3. С. 26-36.

[2] Гохберг И. Ц., Крупник Н. Я. Сингулярные интегральные операторы с кусочно непрерывными коэффициентами и их символы // Изв. АН СССР. Сер. матем. 1971. Т. 35. № 4. C. 940-964.

[3] David G. Opérateurs intégraux singuliers sur certaines courbes du plan complexe // Ann. Sci. École Norm. Sup. (4). 1984. V. 17. P. 157-189.

[4] Дынькин Е. М., Осиленкер Б. П. Весовые оценки сингулярных интегралов и их приложения // Итоги науки и техн. Матем. анализ. Т. 21. М.: ВИНИТИ. С. 42-129.

[5] Böttcher A., Karlovich Yu. I. Toeplitz and singular integral operators on Carleson curves with logarithmic whirl points // Integral Equations Operator Theory. 1995. V. 22. P. 127-161.

[6] Böttcher A., Karlovich Yu. I. Toeplitz and singular integral operators on general Carleson-Jordan curves // Oper. Theory Adv. Appl. 1996. V. 90. P. 119-152.

[7] Karlovich A. Yu. Algebras of singular integral operators with piecewise continuous coefficients on reflexive Orlicz spaces // Math. Nachr. 1996. V. 179. P. 187-222.

[8] Сейфуллаев Р. К. Краевая задача Римана на негладкой разомкнутой кривой // Матем. сб. 1980. Т. 112. № 2. С. 147-161.

[9] Böttcher A., Karlovich Yu. I. Submultiplicativefunctions and spectral theory of Toeplitz operators // Integral Transforms and Special Functions 1996. V. 4. № 1-2. P. 181-202.

[10] Finck T., Roch S., Silbermann B. Two projections theorems and symbol calculus for operators with massive local spectra // Math. Nachr. 1993. V. 162. P. 167-185.

[11] Gohberg I., Krupnik N. Extension theorems for Fredholm and invertibility symbols // Integral Equations Operator Theory. 1993. V. 17. P. 514-529.

[12] Böttcher A., Silbermann B. Analysis of Toeplitz Operators. Berlin: Akademie Verlag, 1989; Berlin-Heidelberg-New York: Springer, 1990.

[13] Красносельский М. А., Рутицкий Я. Б. Выпуклые функции и пространства Орлича. М.: Физматгиз, 1958.

[14] Bennett C., Sharpley R. Interpolation of Operators. London-Boston: Acad. Press, 1988.

[15] Maligranda L. Orlicz Spaces and Interpolation. Sem. Mat. V. 5. Campinas: Univ. Estad. Campinas, 1989.

[16] Matuszewska W., Orlicz W. On certain properties of $\varphi$-functions // Bull. Polish Acad. Sci. Math. Aster. Phys. 1960. V. 8. № 7. P. 439-443.

[17] Maligranda L. Indices and interpolation // Dissertationes Math. 1985. V. 234. P. 1-49.

[18] Boyd D. W. Indices of function spaces and their relationship to interpolation // Canad. J. Math. 1969. V. 21. P. 1245-1254.

[19] Boyd D. W. Indices for the Orlicz spaces // Pacific J. Math. 1971. V. 38. P. 315-323.

[20] Симоненко И. Б. Некоторые обшие вопросы в теории краевой задачи Римана // Изв. АН СССР. Сер. матем. 1968. Т. 32. № 5. С. 1138-1146.

[21] Мусхелишвили Н.И. Сингулярные интегральные уравнения. М.: Наука, 1968.

[22] Гохберг И.Ц., Крупник Н.Я. Системы сингулярных интегральных уравнений в пространствах $L_{p}$ с весом // Докл. АН СССР. 1969. Т. 186. № 5. С. 998-1002.

[23] Спитковский И. М. О блочных операторах и связанных с ними вопросах теории факторизации матриц-функций // Докл. АН СССР. 1980. Т. 254. №4. С. 816-820.

Южно-Украинский педагогический университет им. К. Д. Ушинского,

Поступило

г. Одесса

01.04.97

E-mail: alex@karlik.imem.odessa.ua 